\title{
Inferring power-grid topology in the face of uncertainties
}

\author{
Farnaz Basiri, ${ }^{1}$ Jose Casadiego, ${ }^{2,3}$ Marc Timme, ${ }^{2,3,4}$ and Dirk Witthaut ${ }^{1,5}$ \\ ${ }^{1}$ Forschungszentrum Jülich, Institute for Energy and Climate Research - Systems Analysis and Technology Evaluation (IEK-STE), \\ 52428 Jülich, Germany \\ ${ }^{2}$ Chair for Network Dynamics, Center for Advancing Electronics Dresden (cfaed) and Institute of Theoretical Physics, \\ Technical University of Dresden, 01062 Dresden, Germany \\ ${ }^{3}$ Network Dynamics, Max Planck Institute for Dynamics and Self-Organization (MPIDS), Göttingen 37077, Germany \\ ${ }^{4}$ ETH Zurich Risk Center, 8092 Zurich, Switzerland \\ ${ }^{5}$ Institute for Theoretical Physics, University of Cologne, 50937 Köln, Germany
}

(Received 22 May 2017; revised manuscript received 20 May 2018; published 12 July 2018)

\begin{abstract}
We develop methods to efficiently reconstruct the topology and line parameters of a power grid from the measurement of nodal variables. We propose two compressed sensing algorithms that minimize the amount of necessary measurement resources by exploiting network sparsity, symmetry of connections, and potential prior knowledge about the connectivity. The algorithms are reciprocal to established state estimation methods, where nodal variables are estimated from few measurements given the network structure. Hence, they enable an advanced grid monitoring where both state and structure of a grid are subject to uncertainties or missing information.
\end{abstract}

DOI: 10.1103/PhysRevE.98.012305

\section{INTRODUCTION}

The secure and reliable operation of complex power grids requires a precise knowledge of the grid topology and the state of connected generation and transmission elements. A central tool is "state estimation," i.e., the estimation of the current state of nodes from few, possibly noisy measurements and the grid data. Various methods and algorithms have been developed in this field; see, e.g., [1] for a review. A prime example is the estimation of the voltage phase angles from power flow measurements (see, e.g., [2] for basic textbook examples). State estimation algorithms typically assume that the network structure, i.e., the location and parameters of transmission lines and transformers, are known. However, parts of the information might be lacking or uncertain, for example, for contingencies or attacks, such that the question arises: Is it possible to reconstruct the network structure from local measurements only?

In this article we develop two algorithms that enable the faithful reconstruction of the network structure or parts of it from the measurement of nodal variables via the dc approximation. In particular, we show how to reduce the number of measurements using methods from compressed sensing that exploit the structural properties of power grids. The presented algorithms can find several applications in the monitoring and operation of (smart) power grids. While it is unlikely that the entire network structure is unknown, situations regularly occur where parts of the information is lacking. Manual switches still exist in many distribution grids, transmission lines may fail in contingency cases, and targeted attacks may collapse entire regions of the grid. We present an example where the connectivity in two regions of the grid is a priori unknown, but can be reconstructed from a single snapshot of the nodal variables. In future power grids, the network's structure will become even more variable: The transmission of high-voltage direct-current lines in hybrid power grids can be actively controlled and the effective line parameters can be regulated with flexible alternating-current transmission system devices.

Several methods were previously proposed to deal with missing or erroneous information about the grid topology and parameter [3-17]. These approaches extend the basic idea of state estimation, removing some knowledge about the topology and adding some uncertainties. In contrast this article starts from the reverse case of a completely unknown network, then adding some amount of prior knowledge. Our approach is thus complementary to the existing works such that it can contribute to the development of advanced grid monitoring algorithms $[18,19]$.

The reconstruction schemes proposed in this article extend and adapt previous approaches for network reconstruction developed in different fields of natural science and engineering [20-30]. Recent reviews can be found in [31,32]. The proposed methods extend these approaches; an iterative algorithm is introduced that utilizes the symmetry of the problem and integrates prior knowledge; and we adapt them to the framework of power grid monitoring including a potential combination with standard state estimation. We highlight that typical input data may pose problems for the reconstruction efficiency, which can be partly compensated for by using the iterative recovery algorithm, and discuss the robustness with respect to measurement noise. Finally, we benchmark the proposed method, comparing the efficiency of different algorithms.

\section{STATE AND PARAMETER ESTIMATION}

State estimation extracts the state of an electric power system from a set of measurements [1]. In general, any measurement $z_{i} \in \mathbb{R}$ can be expressed as a function of the system state as

$$
z_{i}=h_{i}(\boldsymbol{x})
$$


where $\boldsymbol{x}$ is the system state and $h_{i}$ is a function specific to the measured quantity $z_{i}$. Assuming we take $M$ measurements, all measurements may be written as

$$
z=h(x),
$$

where $x \in \mathbb{R}^{N \times 1}$ is the system state, $z \in \mathbb{R}^{M \times 1}$ is the vector of measured quantities, and $\boldsymbol{h}: \mathbb{R}^{N} \rightarrow \mathbb{R}^{M}$ is a vector function acting on the measured quantities.

Typically more measurements than the number of state variables to be determined are taken, i.e., $M>N$, such that Eq. (2) is overdetermined. It is then solved in a leastsquares fashion, i.e., one calculates $\boldsymbol{x}$ such that the objective function

$$
J=\|z-\boldsymbol{h}(\boldsymbol{x})\|_{2}^{2}
$$

is minimized. For underdetermined equations (2), typically, many solutions exist.

It is often helpful to work with a simplified dc approximation model when analyzing the inherent limitations of various methods related solely to the measurement configuration [33]. The dc approximation describes the power flow in ac power grids in a linearized way $[2,34-36]$. Specifically, it approximates the real power flow from node $s$ to node $r$ over a transmission line with reactance $X_{s r}$ as

$$
P_{s r}=\frac{\left|V_{s}\right|\left|V_{r}\right|}{X_{s r}} \sin \left(\varphi_{s}-\varphi_{r}\right) \doteq X_{s r}^{-1}\left(\varphi_{s}-\varphi_{r}\right),
$$

where $\varphi_{s}$ is the phase angle at bus $s$. This approximation is to first order in the phase angle difference. Thus, the real power balance at the node $s$ reads

$$
P_{s}=\sum_{r=1}^{N} P_{s r}=\sum_{r=1}^{N} X_{s r}^{-1}\left(\varphi_{s}-\varphi_{r}\right) .
$$

For notational convenience, we summarize these equations in matrix form. We define the vectors of all voltage angles and power injections, $\boldsymbol{\varphi}=\left(\varphi_{1}, \ldots, \varphi_{N}\right)^{\top} \in \mathbb{R}^{N}$ and $\boldsymbol{P}=$ $\left(P_{1}, \ldots, P_{N}\right)^{\top} \in \mathbb{R}^{N}$, where $N$ is the number of buses or nodes in the grid and the superscript $\mathrm{T}$ denotes the transpose operator.

In particular, the dc approximation yields the linear model

$$
\boldsymbol{B} \varphi=\boldsymbol{P},
$$

where the nodal susceptance matrix $\boldsymbol{B} \in \mathbb{R}^{N \times N}$

$$
B_{n k}=\left\{\begin{array}{lll}
\sum_{j=1}^{N} X_{n j}^{-1} & \text { if } & k=n, \\
-X_{n k}^{-1} & \text { if } & k \neq n,
\end{array}\right.
$$

is a Laplacian matrix, which has one zero eigenvalue with eigenvector $(1,1, \ldots, 1)^{\top}$ [37]. This eigenvector represents a global shift of all voltage angles without physical significance. Interestingly, equivalent flow models are used to describe hydraulic networks [38] and vascular networks of plants [39].

Thus in summary, the dc approximation links the state of the grid described by the voltage phase angles $x=\varphi$ to real power flows $\boldsymbol{z}=\boldsymbol{P}$ via the linear function $\boldsymbol{h}=\boldsymbol{B}$. Several extensions of the basic concept of state estimation were developed to deal with missing or erroneous information about the network parameters (see [3] for a review). One branch of methods is based on residual sensitivity analysis.
One solves the conventional least-squares problem (3) and then inspects the residuals $\boldsymbol{z}-\boldsymbol{h}(\boldsymbol{x})$ to reconstruct potential errors [4-7]. Alternatively, one can inspect the Lagrangian multipliers in constrained least-squares optimization $[8,9]$ or minimize the 1-norm [10]. A second branch of methods is based on the augmentation of the state vector. One appends the unknown parameters to the state vector $\boldsymbol{x}$ and then solves a least-squares problem as the one given by Eq. (3) [11-15] or uses a Kalman filter instead $[16,17]$. Both methods can be combined in a two-stage process: First it is detected which network parameters are subject to errors and then the respective parameters are estimated by augmentation.

The installation of new measurement devices and advanced signal processing algorithms enable new methods for power grid monitoring and optimization, see [40] for a review. Topology estimations from phasor measurement data or historical time series were discussed in [41,42], however, without making use of the inherent sparsity of the inference problem. More recently, algorithms were proposed to simultaneously reconstruct the state vector and the topology of a grid and demonstrated for small-scale networks [43,44]. These algorithms rely on power injection data only, but require longer input time series. Topology identification from voltage time series in distribution grids was discussed in [45] and a sparsity-based algorithm for the localization of line outages was presented in [46].

\section{NETWORK RECONSTRUCTION}

In state estimation, one typically assumes that phase angles $\varphi$ are difficult to measure and shall be estimated from the knowledge of $\boldsymbol{B}$ and $\boldsymbol{P}$ in Eq. (6). But we may also reverse the problem and ask what happens if we do not know the nodal susceptance matrix $\boldsymbol{B}$. In particular, can we efficiently reconstruct its entries from measurements of the two remaining quantities $\boldsymbol{P}$ and $\boldsymbol{\varphi}$ ? Such problems may appear hypothetical at first, as the network structure and parameters are generally known. However, there are situations where at least parts of the network structure are unknown: Switches can be open or closed, transmission lines can undergo failures, or plans may be inaccurate. Even more, in times of war or terrorism we may be extremely unsure about the physical integrity of parts of the grid.

To introduce the method, we start from the hypothetical situation where $\boldsymbol{B}$ is completely unknown, whereas $\boldsymbol{P}$ and $\varphi$ are measured perfectly. Related reconstruction problems were pioneered for gene regulatory network [20], oscillator networks [22], and social networks [24]. These articles introduce methods to reconstruct the Laplacian or the adjacency matrix of a network using repeated sampling and exploiting the sparsity of matrices as we will do below. We extend these works by introducing an iterative algorithm that takes into account previous knowledge and can be combined with standard state estimation. The developed algorithms are thoroughly tested for various grids and different input data, including measurement noise. Furthermore, we benchmark the developed method with respect to other algorithms, comparing the amount of input data needed for a faithful reconstruction.

So assume that repeated measurements of $\boldsymbol{P}$ and $\boldsymbol{\varphi}$ are taken at different times $t_{1}, t_{2}, \ldots, t_{M}$. These measurements yield a 
large number of conditions on the entries of $\boldsymbol{B}$,

$$
\sum_{k} B_{r k} \varphi_{k}\left(t_{m}\right)=P_{r}\left(t_{m}\right)
$$

which hold for all rows $r=1, \ldots, N$ of $\boldsymbol{B}$ and all measured time steps $m=1, \ldots, M$ (cf. [14]). Furthermore, we know that the row-sums of the nodal susceptance matrix (7) vanish,

$$
\sum_{k} B_{r k}=0 .
$$

Thus, we have $N(M+1)$ conditions in total, which we can use to reconstruct the unknown entries of $\boldsymbol{B}$.

\section{A. Row-wise reconstruction}

In a first approach we aim to reconstruct the nodal admittance matrix row by row (cf. $[22,26,32,47])$. To simplify notation we collect all measurement conditions (8) and the condition (9) for a given row $r$ and rewrite them in matrix form

$$
\underbrace{\left(\begin{array}{ccc}
\varphi_{1}\left(t_{1}\right) & \cdots & \varphi_{N}\left(t_{1}\right) \\
\vdots & & \vdots \\
\varphi_{1}\left(t_{M}\right) & \cdots & \varphi_{N}\left(t_{M}\right) \\
1 & \cdots & 1
\end{array}\right)}_{=: \boldsymbol{\Phi}} \underbrace{\left(\begin{array}{c}
B_{r 1} \\
\vdots \\
B_{r N}
\end{array}\right)}_{=: \boldsymbol{B}_{r}}=\underbrace{\left(\begin{array}{c}
P_{r}\left(t_{1}\right) \\
\vdots \\
P_{r}\left(t_{M}\right) \\
0
\end{array}\right)}_{=: \mathcal{P}_{r}} .
$$

Here, $\boldsymbol{B}_{r} \in \mathbb{R}^{N \times 1}$ denotes the transpose of the $r$ th row of the nodal admittance matrix $\boldsymbol{B}$. The matrix $\boldsymbol{\Phi} \in \mathbb{R}^{(M+1) \times N}$ summarizes all measured phase angles at all time steps, the vector $\mathcal{P}_{r} \in \mathbb{R}^{(M+1) \times 1}$ includes all power injections for node $r$ only, and the last row of the matrix equation represents condition (9).

If the system of equations (10) is overdetermined, $M+$ $1>N, \boldsymbol{B}_{r}$ is easily reconstructed via least squares [32,47] minimizing

$$
\left\|\boldsymbol{\Phi} \boldsymbol{B}_{r}-\mathcal{P}_{r}\right\|_{2}^{2}=\sum_{k=1}^{M+1}\left(\boldsymbol{\Phi} \boldsymbol{B}_{r}-\mathcal{P}_{r}\right)_{k}^{2} .
$$

To efficiently reconstruct the grid topology, we want to rely on as few measurements as possible. If $M+1<N$, Eq. (10) is underdetermined, and thereby may admit several solutions [32]. So, is it possible to obtain the correct solution also in this case? Do we have more information about $\boldsymbol{B}_{r}$ which we can exploit? Indeed, we know that a power grid is typically very sparse; a single substation is connected to only a few other substations. Thus we choose the one solution to Eq. (10) which minimizes

$$
\left\|\boldsymbol{B}_{r}\right\|_{0}=\lim _{p \rightarrow 0}\left(\sum_{k=1}^{N}\left|B_{r k}\right|^{p}\right)^{1 / p} .
$$

We use the notation $\|\cdot\|_{0}$ for the sake of convenience, noting that this is not a vector norm in the strict sense. Unfortunately, the direct minimization is computationally hard in general. In 2006, Donoho [48] and Candes et al. [49] showed that an efficient reconstruction is nevertheless possible using a convex surrogate for sparsity: the 1-norm. Under weak conditions, the correct sparse solution can be calculated efficiently by minimizing the 1-norm

$$
\left\|\boldsymbol{B}_{r}\right\|_{1}=\sum_{k=1}^{N}\left|\boldsymbol{B}_{r k}\right|
$$

subject to the constraint (10).

\section{B. Partial reconstruction}

In a typical application we know the value of $B_{r s}$ of all transmission lines $(s, r)$ in one part of the grid and have to reconstruct only the remaining entries of the matrix $\boldsymbol{B}$; for instance, we might want to monitor the position of manual switches at remote places of the grid.

To keep track of our knowledge about the network, we define the matrix $\boldsymbol{K} \in \mathbb{R}^{N \times N}$ with entries

$$
K_{r s}:= \begin{cases}1 & \text { if the value of } B_{r s} \text { is known for line }(r, s) \\ 0 & \text { otherwise. }\end{cases}
$$

For the $r$ th row we reduce Eq. (10) to

$$
\boldsymbol{\Phi}_{r}^{\mathrm{red}} \boldsymbol{B}_{r}^{\mathrm{red}}=\mathcal{P}_{r}^{\mathrm{red}},
$$

where

$$
\mathcal{P}_{r}^{\mathrm{red}}=\mathcal{P}_{r}-\sum_{c=1}^{N} B_{r c} K_{r c}\left(\begin{array}{c}
\varphi_{c}\left(t_{1}\right) \\
\vdots \\
\varphi_{c}\left(t_{M}\right) \\
1
\end{array}\right)
$$

and $\boldsymbol{\Phi}_{r}^{\text {red }}$ is the submatrix of $\boldsymbol{\Phi}_{r}$ obtained by deleting all columns $c$ for which $K_{r c}=1$ and $\boldsymbol{B}_{r}^{\text {red }}$ is the submatrix of $\boldsymbol{B}_{r}$ obtained by deleting all rows $r^{\prime}$ for which $K_{r, r^{\prime}}=1$. The dimension of the reduced linear system of equations (15) is smaller than in the original problem (10), such that the correct solution can typically be found from fewer measurements.

\section{Positivity of line susceptances}

The reconstruction methods discussed above rely on the utilization of structural information about the matrix $\boldsymbol{B}$, in particular its sparsity. For transmission lines we know that the reactance $X_{r s}>0$ such that even more prior structural information is available. To boost the reconstruction algorithms we can thus add the constraint

$$
B_{r s} \leqslant 0 \quad \text { for all pairs } r \neq s
$$

to the minimization of the 1-norm $\left\|\boldsymbol{B}_{r}\right\|_{1}$.

However, care has to be taken as the sign constraint does not always hold. Branches with $X_{s r}<0$ can occur in the modeling of two-winding tap-changing transformers and three-winding transformers. Hence the sign constraint (17) can be employed only if no such elements are present in the grid or if we know their location. In the following we consider both cases with and without imposing the sign constraint (17) when testing the proposed algorithms. In particular, one test grid (the IEEE 300 bus test grid) contains one negative reactance branch, whose location is assumed to be known such that we never impose the constraint (17) for this branch. 


\section{Reconstruction from power flow measurements}

The reconstruction scheme introduced above requires the knowledge of the voltage phase angle at all nodes of the grid. These data can in principle be obtained using phasor measurement units, but these are typically very expensive. The measurement of other quantities such as real power flows is typically much simpler.

Fortunately, we usually do not have to reconstruct the entire grid in most cases. In a typical application we know a lot about the grid and have to reconstruct only parts of the matrix $\boldsymbol{B}$. Then the measurement of power injections and real power flows of a certain subset of transmission lines can be enough to perform the reconstruction of the remaining data using the methods described above. Technically, this amounts to combining methods of classical state estimation and network reconstruction.

So assume that we can measure the power injection $P_{n}$ for all nodes $n \in\{1, \ldots, N\}$ at the time steps $t_{1}, \ldots, t_{M}$. In addition we have knowledge about the transmission line parameters, in particular $X_{s r}$, and measure the real power flow

$$
P_{s r}\left(t_{m}\right)=X_{s r}^{-1}\left[\varphi_{r}\left(t_{m}\right)-\varphi_{s}\left(t_{m}\right)\right]
$$

for a subset $\mathcal{L}$ of all transmission lines at all time steps $t_{1}, \ldots, t_{M}$. If this set of equations is fully determined or even overdetermined we reconstruct the state vector $\varphi\left(t_{m}\right)$ as in the classical state estimation problem described in Sec. II. As before, the resulting estimates for the nodal phase angles for all time steps $t_{1}, \ldots, t_{M}$ are then summarized in the matrix $\boldsymbol{\Phi}$. In addition we have partial knowledge about the nodal susceptance matrix, in particular we already know the entries

$$
B_{r s}=B_{s r}=-X_{s r}^{-1}
$$

for all $(s, r) \in \mathcal{L}$. This information can then be used to reconstruct the remaining entries of $\boldsymbol{B}$ as described in the previous section.

\section{IMPLEMENTATION}

\section{A. Row-wise reconstruction}

The row-wise reconstruction problem introduced in Sec. III A is solved using the algorithm depicted in Fig. 1. Source code for the central part of the program code is listed in Fig. 2.

First data are collected and the matrix $\boldsymbol{\Phi}$ and the vectors $\mathcal{P}_{r}$ are formed. Then the problem is solved depending on the rank of the matrix $\boldsymbol{\Phi}$. If the system is (over)determined, we directly solve it for $\boldsymbol{B}$ using the MATLAB function mldivide. If the system is underdetermined we proceed row by row and reconstruct $\boldsymbol{B}_{r}$ by solving $\min \left\|\boldsymbol{B}_{r}\right\|_{1}$ s.t. $\boldsymbol{\Phi} \boldsymbol{B}_{r}=\mathcal{P}_{r}$. This optimization problem can be mapped to a linear program as described in Appendix B and solved with the MATLAB function linprog.

The code listed in Fig. 2 illustrates the reconstruction algorithm without utilizing the sign constraint (17). This constraint can easily be added to the code as described in Appendix B.

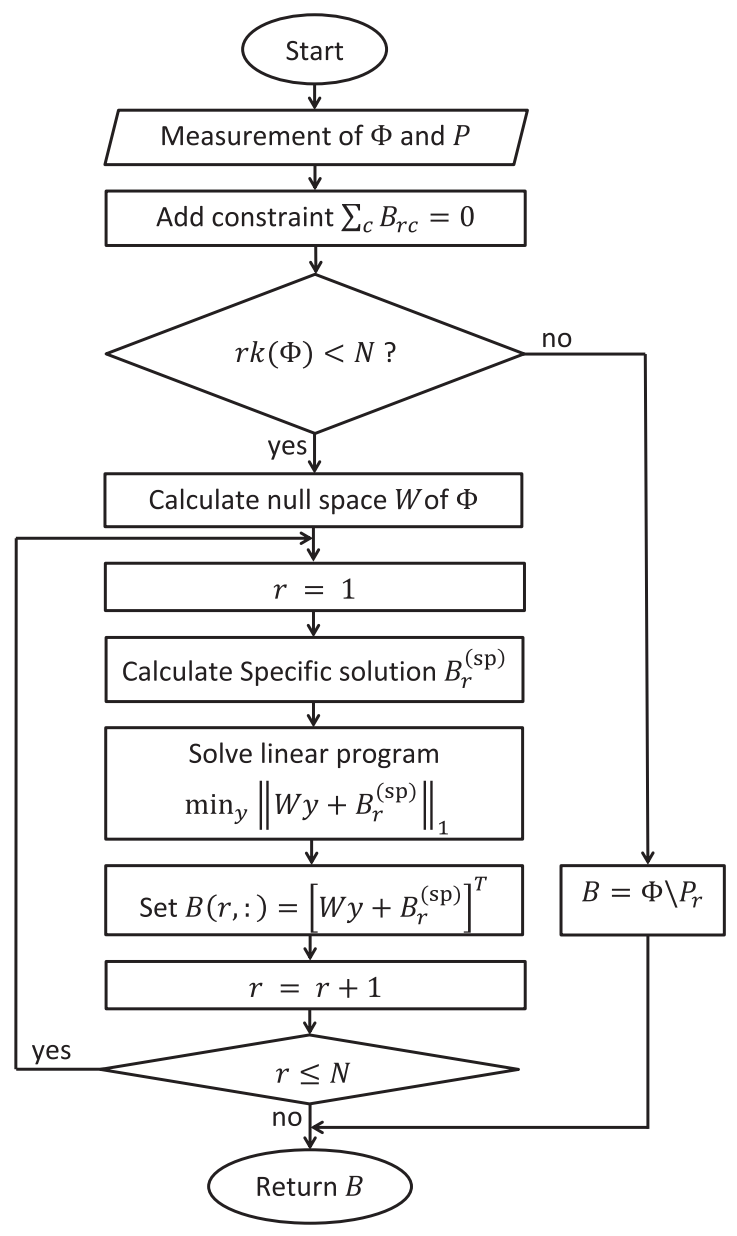

FIG. 1. Flow chart for row-wise reconstruction without implementing the sign constraint (17). MATLAB code for the central part is listed in Fig. 2.

\section{B. Iterative reconstruction}

The reconstruction problem with prior knowledge introduced in Sec. IIIB is solved using the algorithm depicted in Fig. 3. Most interestingly, we can also use this algorithm to greatly improve the convergence of the reconstruction algorithm. For intermediate values of $M$ we typically face the situation that some rows are successfully reconstructed while others are not. If we have not reconstructed $\boldsymbol{B}$ successfully, but gained further knowledge, we may use it in the following. Having successfully reconstructed the $r$ th row of $\boldsymbol{B}$, we also know the entries $B_{r^{\prime}, r}=B_{r, r^{\prime}}$ for all other rows $r^{\prime}$ due to the symmetry of the matrix. We thus propose to perform the reconstruction iteratively. In each step of the reconstruction process we use the initial knowledge about the entries of the matrix $\boldsymbol{B}$ and also the knowledge gained in previous steps.

The algorithm shown in Fig. 3 starts with the input of the measurement results and the prior knowledge of the system which is encoded in the matrices $\boldsymbol{B}$ and $\boldsymbol{K}$. It solves the reconstruction problem iteratively, processing row by row in each iteration. For each row, we calculate the reduced matrices $\boldsymbol{\Phi}_{r}^{\text {red }}$ and $\mathcal{P}_{r}^{\text {red }}$, see Eq. (15) and then attempt to reconstruct the vector $\boldsymbol{B}_{r}^{r}$ ad as described above. If the reconstruction was successful, we add the results to the matrix $\boldsymbol{B}$ and update the 


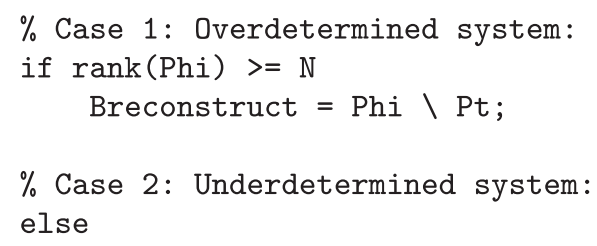

FIG. 2. MATLAB code for row-wise reconstruction.

knowledge matrix $\boldsymbol{K}$. To facilitate the bookkeeping we define two matrices $\boldsymbol{K}$ and $\boldsymbol{K}^{\text {new }}$ : We set $\boldsymbol{K}=\boldsymbol{K}^{\text {new }}$ at the beginning of each iteration and only modify $\boldsymbol{K}^{\text {new }}$ during the step. The iteration stops when the reconstruction has been successfully completed, i.e., $K_{r c}^{\text {new }}=1$ for all $r, c=\{1 \ldots, N\}$, or when no further progress has been made, i.e., $K^{\text {new }}$ has not been modified during the last step.

One unsolved problem remains: If we do not know the matrix $\boldsymbol{B}$ a priori, how can we know if the reconstruction of the $r$ th row has been successful? In the underdetermined case we cannot decide whether the reconstructed values $B_{r c}$ are definitely correct, but we can decide if they are reasonable in terms of the connectivity of the grid, i.e., if the number of nonzero entries in $\boldsymbol{B}_{r}$ is as small as expected. This partly rules out undesired solutions, where minimization of the 1norm (13) does not yield sufficiently sparse solutions. In the algorithm shown in Fig. 3 we thus adopt the definition that the reconstruction of the $r$ th row is assumed to be successful if (a) the linear system is overdetermined or $(b)$ the reconstructed row $\boldsymbol{B}_{r}$ is sufficiently sparse, i.e., $\sum_{j} H\left(\left|B_{r j}\right|\right) \leqslant d_{\max }$ for some upper limit $d_{\max }$ and $H(x)$ being the Heaviside step function. This upper limit is chosen the same for all rows and must be larger or equal to the maximum degree of any node in the grid. Based on experience and on typical degrees of real power grids, we use a value $d_{\max }=15$ in what follows.

A slight adaption of this criterion is necessary if the measurements of the phases or power injections are subject to noise. Then the reconstructed vector $\boldsymbol{B}_{r}$ will also be noisy;

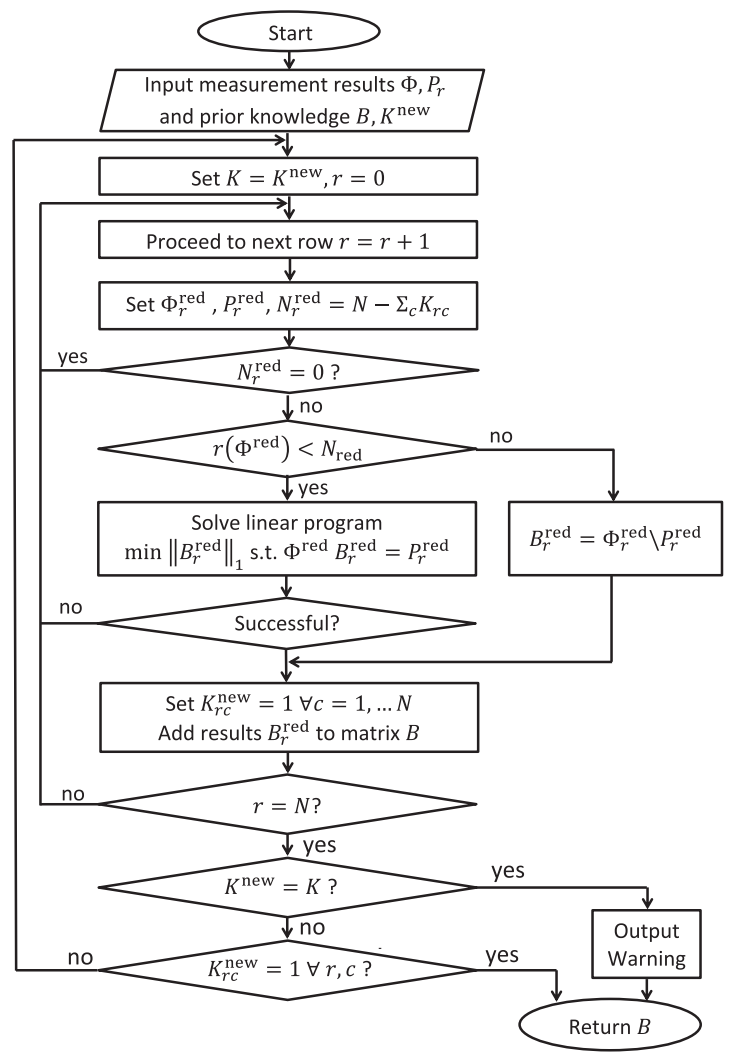

FIG. 3. Flow chart for the iterative reconstruction algorithm without implementing the sign constraint (17).

in particular, it will not be strictly but only approximately sparse. Thus we relax the above criterion for the acceptance of a reconstructed row counting only entries whose magnitude exceed a certain threshold

$$
\text { number of entries }=\sum_{j} H\left(\left|B_{r j}\right|-\epsilon^{\prime}\right)
$$

for some $\epsilon^{\prime}>0$. The criterion (2) for the acceptance of a reconstructed row then reads $\sum_{j} H\left(\left|B_{r j}\right|-\epsilon^{\prime}\right) \leqslant d_{\max }$.

\section{APPLICATIONS AND PERFORMANCE}

\section{A. Reconstruction from time series}

We demonstrate the applicability of network reconstruction from time series measurements for a test grid taken from [50] illustrated in Fig. 4(a). This data set includes hourly data for demand and generation $P_{k}(t)$ for one year and all $N=117$ nodes. The nodal voltage angles $\varphi_{k}(t)$ are then obtained by solving the DC approximation (6) for all time steps. The resulting time series of the power injections and angles are shown in Fig. 4 (b) for two nodes as an example.

The algorithm shown in Fig. 3 can now reconstruct the entire network topology, i.e., all entries of the nodal susceptance matrix $\boldsymbol{B}$, from the time series data. To evaluate the performance of the algorithm we vary the amount of input data. For each value of $M$ we run the algorithm and count the number of successfully reconstructed entries and the number of reconstruction errors (those entries that do not match the ground truth). To account for small numerical errors, we say 

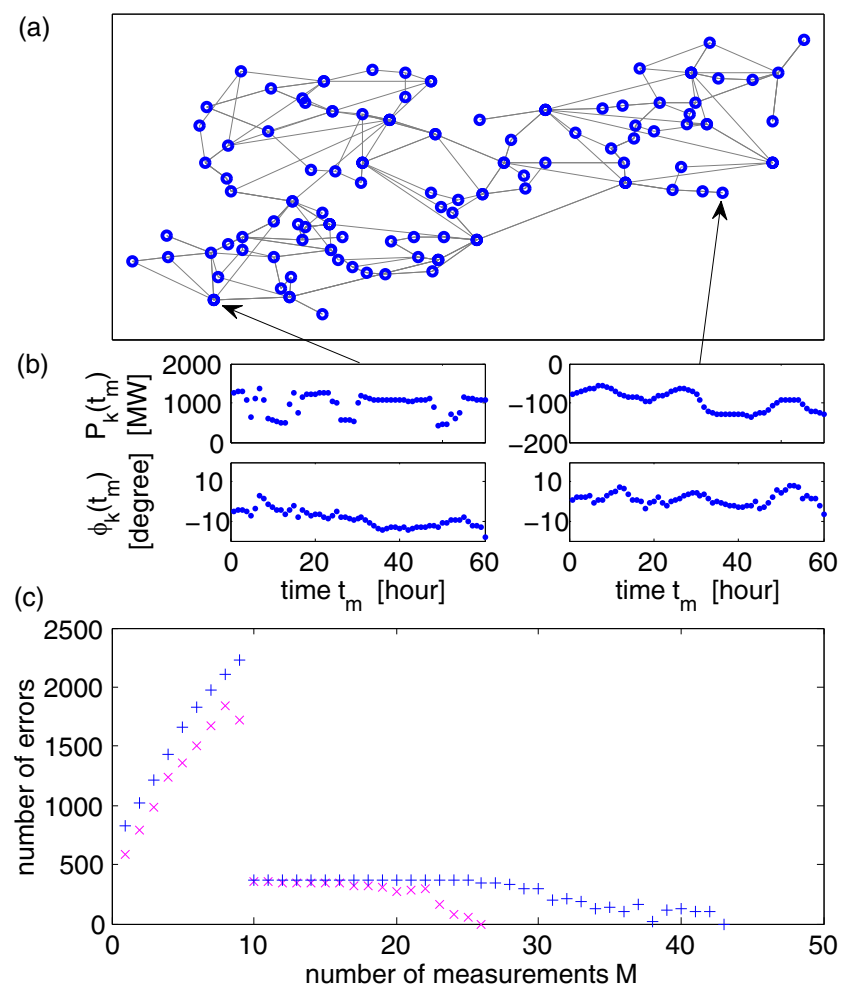

FIG. 4. Example of the complete grid reconstruction for a test grid with $N=117$ nodes. (a) Test grid from [50]. It is assumed that the network topology, i.e., the location and parameters of the transmission lines (grey) are completely unknown. (b) The reconstruction is based on the time series of the nodal power injections $P_{k}(t)$ and voltage angles $\varphi_{k}(t)$. Two time series are shown. (c) Performance of the iterative algorithm shown in Fig. 3 taking into account the sign constraint (+) or discarding it $(\times)$. Perfect reconstruction is obtained with $M \geqslant 26$ measurements when the sign constraint is implemented. We use the test grid and the load and generation time series of the scenario 2013 from [50]. For the sake of simplicity we have removed the import and export nodes and rescaled the generation to exactly match the load.

that a link $(r, k)$ is successfully reconstructed if

$$
\left|B_{r, k}^{\text {recon }}-B_{r, k}^{\text {true }}\right|<\epsilon\left|B_{r, k}^{\text {true }}\right| .
$$

The absence of a line between two nodes $r$ and $k$ is successfully reconstructed if

$$
\left|B_{r, k}^{\text {recon }}\right|<\epsilon \min _{i \neq j}\left|B_{i, j}^{\text {true }}\right|
$$

where the numerical tolerance is chosen as $\epsilon=10^{-3}$.

Figure 4(c) shows that $M_{\min }=26$ measurements are sufficient to faithfully reconstruct the entire nodal susceptance matrix $\boldsymbol{B}$ using the iterative algorithm and including the sign constraint (17). Most importantly, a faithful reconstruction is possible in the strongly underdetermined case: The minimum number of measurements $M_{\min }=26$ is less than a quarter of the matrix dimension $N=117$. This is possible because we can exploit the sparsity of matrix $\boldsymbol{B}$ as an additional structural information.

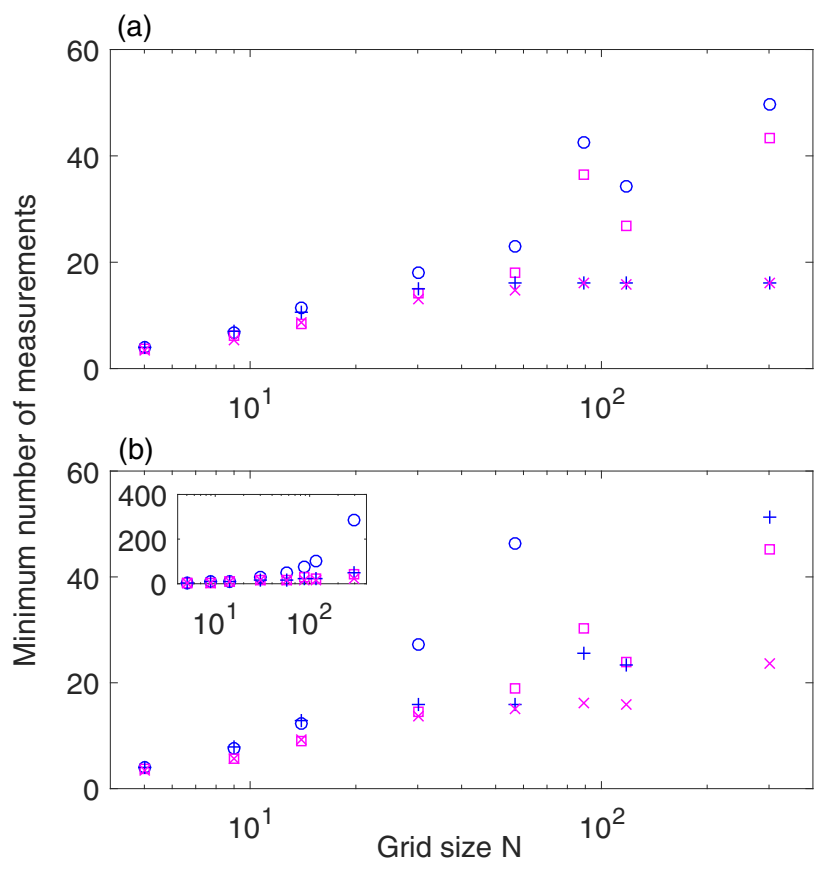

FIG. 5. Performance of the proposed algorithms for power grid reconstruction for various test grids taken from [51,52]. We use synthetic power injection data, choosing either (a) the $\varphi_{k}$ randomly (type I) or (b) the $P_{k}$ randomly (type II). Shown is the minimum number of measurements $M$ needed to obtain a faithful reconstruction for the row-wise reconstruction algorithm with $(\square)$ and without (o) sign constraint and the iterative algorithm with $(x)$ and without $(+)$ sign constraint. Results have been averaged over 10 random realizations.

\section{B. Minimum measurement requirements and scaling}

The algorithms presented in Figs. 1 and 3 allow a reconstruction of the network topology also in the underdetermined case. But how many measurements $M_{\text {min }}$ are needed for a faithful reconstruction and how does this number scale with the grid size $N$ ?

To systematically study the efficiency of the reconstruction algorithms, we consider various test grids of different sizes taken from [51,52] and use randomized synthetic data for the time series. We consider two different types of time series data. First we draw the voltage phase angles $\varphi_{k}(t)$ uniformly at random from the interval $[-\pi / 8,+\pi / 8]$ (type I). This scenario is not realistic but close to the original mathematical work $[48,49]$, where random Gaussian sampling vectors are considered. Second, we choose the power injections $P_{k}(t)$ at random from a normal distribution with mean zero and standard deviation as in the original test grid (type II). For both types and both reconstruction algorithms (row-wise and iterative), we run the reconstruction algorithm as a function of the number of measurements $M$ taken into account and determine the minimum number of measurements $M_{\min }$ for a faithful reconstruction, i.e., zero reconstruction errors. Each numerical experiment is repeated 10 times. Results are shown in Fig. 5.

First we observe that an efficient reconstruction is possible with both algorithms for the type I input data as shown in Fig. 5(a). The minimum number of measurements $M_{\min }$ is much smaller than the grid size $N$ in all cases. The simple 

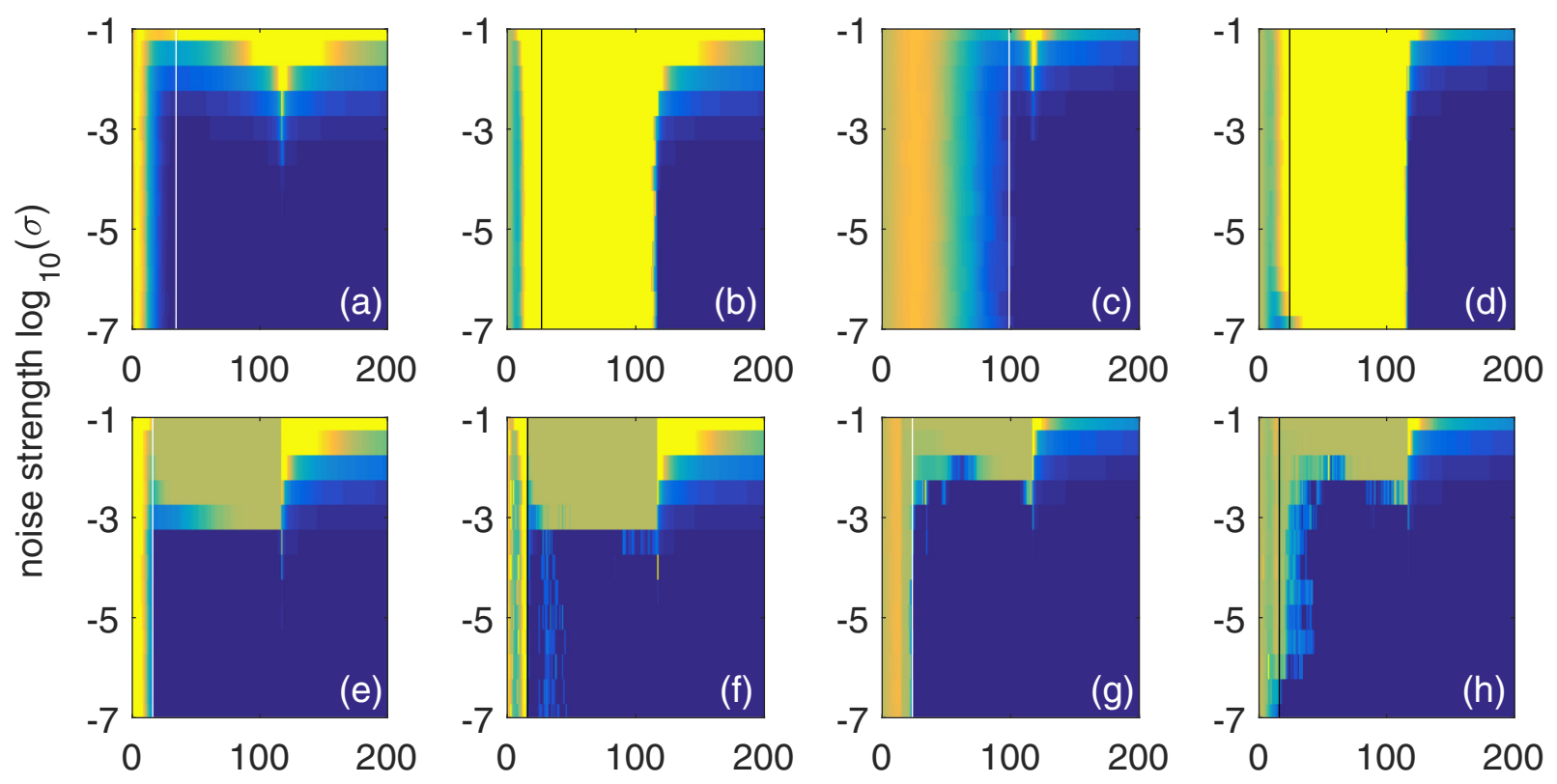

error $\mathrm{E}$
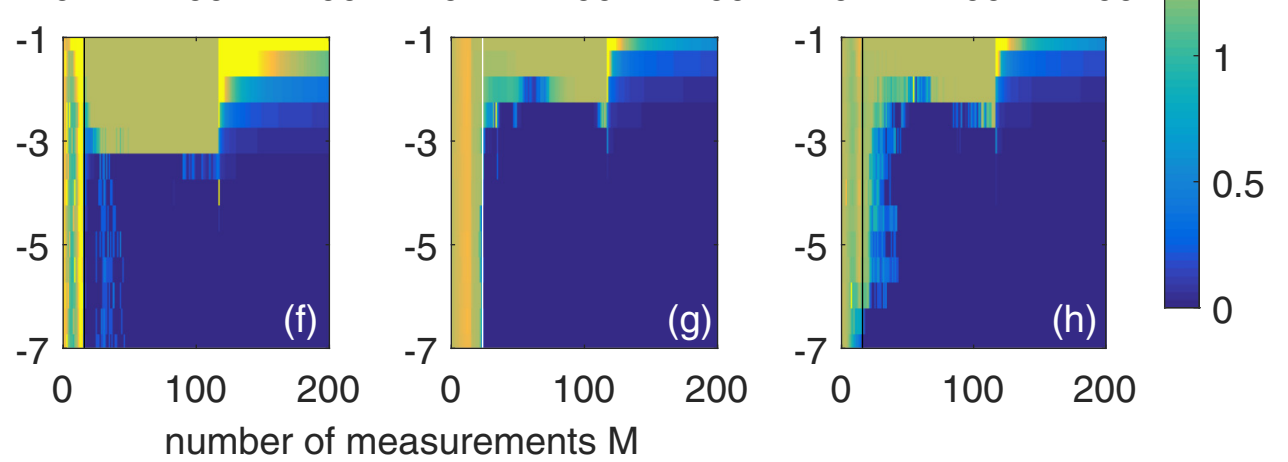

FIG. 6. Network reconstruction in the presence of measurement noise. The color map plots show the reconstruction error [Eq. (24)] as a function of the number of measurement snapshots $M$ and the noise strength, assuming that the phase measurements are subject to independent Gaussian noise with standard deviation $\sigma$. The white and black lines indicate the minimum number of measurements $M_{\text {min }}$ reported in Fig. 5 . Results are shown in (a)-(d) for the row-based algorithm and in (e)-(h) for the iterative algorithm. We run the reconstruction both without implementing the sign constraint (17), see panels (a), (c), (e), (g); and with the sign constraint (17), see panels (b), (d), (f), (h). The algorithms are tested for the IEEE 118 bus test grid taken from [51] for synthetic power injection data, choosing either the $\varphi_{k}$ randomly [type I data, panels (a), (b), (e), (f)] or the $P_{k}$ [type II data, panels (c), (d), (g), (h)] randomly. Results have been averaged over five random runs.

row-wise algorithm performs less well because it does not exploit the symmetry of the matrix $\boldsymbol{B}$, such that $M_{\min }$ is larger by a factor of up to 3 . Furthermore, implementing the sign constraint (17) only has a minor influence on the performance of the reconstruction for type I input data.

The theory of compressed sensing shows that a reconstruction of sparse vectors is possible with high probability from a number of measurements scaling only logarithmically with the problem dimension $N$ if some conditions are satisfied [48,49]. This logarithmic scaling is well confirmed by the results of our numerical experiments as observed in Fig. 5(a). For the iterative algorithm, the scaling appears to be even more favorable.

A different result emerges for type II input data as shown in Fig. 5(b). The proofs for the efficiency of compressed sensing depend on two features of the sampling vectors: isotropy and incoherence. Roughly speaking, isotropy means that all directions in the vector space are sampled equally well. Incoherence guarantees that the redundancy in the information gained by two measurements is small. Both features are no longer guaranteed for type II input data. In fact, we observe that the simple row-wise reconstruction algorithm without sign constraint does not perform well in this case. The minimum number of measurements $M_{\min }$ is only slightly smaller than the problem dimension $N$. The logarithmic scaling is lost.

Nevertheless we find that an efficient reconstruction is still possible if we utilize further structural information. Implementing the sign constraint (17) in the row-wise algorithm drastically reduces the minimum number of measurements $M_{\min }$. Exploiting the symmetry of matrix $\boldsymbol{B}$ in the iterative algorithm is even more effective. An efficient reconstruction from few measurements is possible and the logarithmic scaling of $M_{\min }$ with the problem size $N$ appears to be restored. Adding the sign constraint further reduces $M_{\min }$ by a factor of up to 2.3 .

\section{Robustness to noise}

Every real measurement is susceptible to noise. Hence, a reconstruction algorithm is applicable in reality only if it is robust at least for weak noise. We evaluate the performance of the two algorithms described above assuming that the measured phases differ from the true values

$$
\varphi_{j}^{\text {meas }}\left(t_{m}\right)=\varphi_{j}^{\text {true }}\left(t_{m}\right)+e_{j, m},
$$

We assume that the measurement errors $e_{j, m}$ are independent and follow a normal distribution with mean 0 and standard deviation $\sigma$. The quality of the reconstruction of the matrix $\boldsymbol{B}$ is evaluated by the sum of absolute deviations from the true value,

$$
E=\sum_{r, k=1}^{N}\left|B_{r k}^{\mathrm{recon}}-B_{r k}^{\mathrm{true}}\right| .
$$

We evaluate the impact of noise for the IEEE 118 bus test grid for different randomized input data (types I and II) comparing the row-wise and iterative algorithms both with and without implementing the sign constraint (17).

Numerical results reported in Fig. 6 show that both algorithms are robust to weak noise if the sign constraint (17) is discarded. Significant deviations from the noiseless case 
are found only if the standard deviation exceeds a threshold $\sigma \geqslant 10^{-4}$. In particular the measurement error $E$ is close to zero if the number of measurement snapshots $M$ exceeds the minimum number $M_{\text {min }}$ determined for the noiseless case (see Fig. 5) and $\sigma \leqslant 10^{-4}$.

In contrast, the sign constraint (17) can make the reconstruction highly susceptible to measurement noise. In particular, the row-wise algorithm fails already for a very weak noise level $\sigma=10^{-7}$ regardless of the input data (types I or II). The iterative algorithm is less susceptible, because it implements a test of every reconstruction result in view of its sparsity. This test is capable of avoiding the massive failures present in the row-wise algorithm to a large extent. Still, the minimum number of measurements for a faithful reconstruction increases already for weak noise levels. We conclude that the implementation of the sign constraint (17) is not always beneficial because it reduces the robustness of the algorithm.

\section{Comparison to other sparse signal recovery methods}

The proposed algorithms for network reconstruction are based on convex relaxation: A sparse solution to an underdetermined system of equations is found by minimizing the 1-norm [Eq. (13)]. Several alternatives have been proposed for this task; see [53] for an introductory review.

We compare the efficiency of different methods for the network reconstruction to provide a benchmark for the two proposed algorithms. To this end, we replace the 1-norm minimization in the network reconstruction algorithm (lines 11-20 in Fig. 2) by two other methods: the orthogonal matching pursuit (OMP) [54] and the orthogonal least squares (OLS) [55]. OLS requires us to specify a maximum target sparsity of a vector beforehand, which we choose as $d_{\max }+1$. We then repeat simulation for several test grids and evaluate the minimum number of measurements needed for a faithful reconstruction of matrix $\boldsymbol{B}$. We discard the sign constraint (17) since it degrades the robustness as discussed above. The results are displayed in Fig. 7.

For the row-wise reconstruction we observe no significant difference between the minimization of the 1-norm, OMP, and OLS. In all cases the reconstruction remains rather inefficient for type II input data, due to the lack of incoherence and isotropy of the sampling vectors discussed above. Vast differences between the methods are observed for the iterative reconstruction algorithm for both types of input data. The minimization of the 1-norm outperforms all alternatives by far: For the largest test grid $(N=300)$, the minimum number of measurements for a faithful reconstruction is smaller than for the alternative methods by a factor of more than 2.8 (type I) or 6.1 (type II).

\section{E. Reconstruction from flow measurements}

The iterative algorithm allows us to take into account prior knowledge to efficiently reconstruct the missing information about the grid topology. Such a problem can arise in practice when a larger part of the grid is subject to damages or attacks. An example of such a situation is depicted in Fig. 8(a). It is assumed that we have no reliable information about the grid in two areas indicated by the question marks. However, we
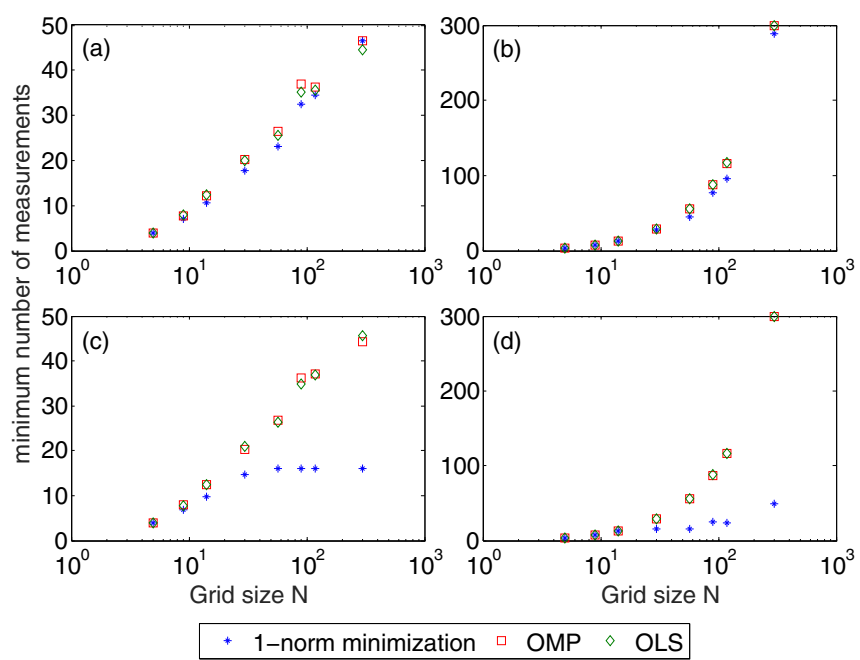

FIG. 7. Comparison of three different sparse signal recovery methods for network reconstruction: the 1-norm minimization, the orthogonal matching pursuit (OMP) [54], and the orthogonal leastsquares (OLS) [55]. We evaluate the minimum number of measurements for a faithful reconstruction for several test grids of increasing size $N$ and different input data (type I vs II). (a),(b) Using the row-wise reconstruction algorithm no significant differences are observed. (c),(d) Using the iterative reconstruction algorithm, 1-norm minimization outperforms the alternative methods by far. In all cases, the reconstruction is performed without enforcing the sign constraint (17).

do have information about the connections of all remaining nodes and we assume that we can measure the real power flow along the colored solid lines. The prior knowledge of
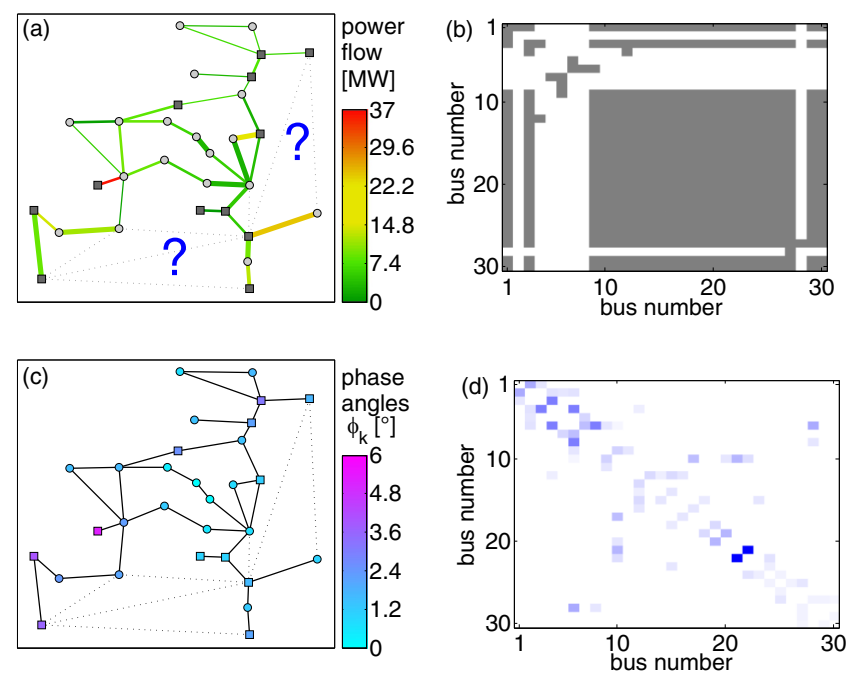

FIG. 8. Reconstruction of missing information from flow measurements. (a) The IEEE 30-bus test grid. Real power flows are measured on the colored lines. In two regions the grid topology is assumed to be unknown. (b) The prior knowledge matrix $K$. Grey shading indicates that the respective line parameter $B_{j k}$ is known. The white region is completely unknown. (c) The voltage phase angles $\varphi_{k}$ are reconstructed from the power flow measurements using standard state estimation methods. (d) The complete grid topology is perfectly reconstructed using the algorithm shown in Fig. 3. 
the grid topology is encoded in matrix $\boldsymbol{K}$, which is illustrated in Fig. 8(b). White entries indicate where we do not know the entries of matrix $\boldsymbol{B}$.

The missing information is found by combining classical state estimation and network reconstruction. First, the voltage phase angles for all nodes of the network are reconstructed as described in Sec. II, the results being shown in Fig. 8(c). Second, the iterative reconstruction algorithm shown in Fig. 3 is applied using the measured power injections, the prior knowledge $\boldsymbol{B}$ and $\boldsymbol{K}$ as well as the estimated voltage phase angles as input data. The algorithm then faithfully reconstructs all the missing information about the grid topology. The reconstructed values of the line susceptances $X_{s r}^{-1}$ shown in Fig. 8(d) exactly match the true values. Most importantly, the full reconstruction is possible already for $M=1$ in this case, i.e., with a single snapshot of the power injections $\boldsymbol{P}$, making use of the prior knowledge.

\section{CONCLUSION}

We have introduced two algorithms to reconstruct the structure of a power grid from nodal measurements only. One algorithm is straightforward and row-based, reconstructing the lines of the grid node by node. The second is iterative and takes into account both prior knowledge about the presence of absence of lines as well as the knowledge generated by that algorithm during previous steps. We have demonstrated how these algorithms can be used to reconstruct the entire network structure from time series or missing information on the grid topology from a single snapshot.

The presented algorithms exploit several structural properties of power grids to reduce the number of necessary measurements. Power grids are typically very sparse, i.e., each substation is connected to only a few other substations. Hence, methods from compressed sensing can be used to allow faithful reconstructions also in the underdetermined case. The iterative reconstruction algorithm makes use of the symmetry of the nodal susceptance matrix. Loosely speaking the algorithm solves the simple parts of the reconstruction problem first and then uses the gained information for the remaining parts. We have shown that this trick leads to a vast reduction of measurement resources.

\section{ACKNOWLEDGMENTS}

We thank D. Gross and R. Kueng for inspiring discussions. We gratefully acknowledge support from the Helmholtz Association (via the joint initiative "Energy System 2050 - A Contribution of the Research Field Energy" and the Grant No. VH-NG-1025 to D.W.), the Federal Ministry of Education and Research (BMBF Grant No. 03SF0472A-F to D.W. and M.T.), and the German Science Foundation (DFG) by a grant toward the Center of Excellence "Center for Advancing Electronics Dresden" (cfaed).

\section{APPENDIX A: LEAST-SQUARES SOLUTION}

If the system of equations (10) is overdetermined, it then must be solved in a least-squares fashion, i.e., we have to solve

$$
\min _{\boldsymbol{B}_{r}}\left\|\boldsymbol{\Phi} \boldsymbol{B}_{r}-\mathcal{P}_{r}\right\|_{2}^{2},
$$

which leads to [32]

$$
\boldsymbol{B}_{r}=\left(\boldsymbol{\Phi}^{T} \boldsymbol{\Phi}\right)^{-1} \boldsymbol{\Phi}^{T} \mathcal{P}_{r}
$$

provided that $\boldsymbol{\Phi}$ is full rank such that the inverse exists. Leastsquares solutions are already implemented in many numerical solvers, for instance in the MATLAB function mldivide.

\section{APPENDIX B: MINIMIZING THE 1-NORM}

The key to an efficient reconstruction of $\boldsymbol{B}$ from an underdetermined system of equations is the minimization of the 1-norm $[48,49]$. This problem can be mapped to a linear program which can be solved efficiently, i.e., the computation time scales at most polynomially with the input length.

So we consider the linear system of equations (10) and assume that it is solvable and underdetermined. The solutions span an affine subspace of $\mathbb{R}^{N}$ with dimension $D=N-$ $\operatorname{rank}(\boldsymbol{\Phi})$. All solutions can be written as

$$
\boldsymbol{B}_{r}=\boldsymbol{B}_{r}^{(\mathrm{sp})}+\boldsymbol{W} \boldsymbol{y}
$$

where the columns of the matrix $\boldsymbol{W} \in \mathbb{R}^{N \times D}$ form a basis for the kernel (null space) of $\boldsymbol{\Phi}$ and $\boldsymbol{y} \in \mathbb{R}^{D}$ is a vector of parameters. $\boldsymbol{B}_{r}^{(\mathrm{sp})}$ is a specific solution to Eq. (10); in the implementation it is obtained using the MATLAB function mldivide. We now search for the vector $\boldsymbol{y}$ such that the 1-norm (13) assumes its minimum. This optimization problem is rewritten as a linear program [56]

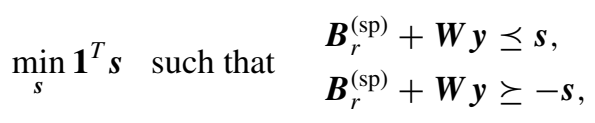

where $s \in \mathbb{R}^{N}$ is an auxiliary variable, $\mathbf{1} \in \mathbb{R}^{N}$ is a vector of ones, and $\preceq$ and $\succeq$ denote entry-wise comparison. In the standard form of many commercial solvers the optimization problem reads

$$
\min _{\boldsymbol{x}} \boldsymbol{f}^{T} \boldsymbol{x} \text { such that } \boldsymbol{A x} \preceq \boldsymbol{b},
$$

using the further auxiliary variables

$$
\begin{aligned}
& \boldsymbol{x}=\left(\begin{array}{c}
\boldsymbol{y} \\
\boldsymbol{s}
\end{array}\right), \quad \boldsymbol{A}=\left(\begin{array}{cc}
\boldsymbol{W} & -\mathbb{1} \\
-\boldsymbol{W} & -\mathbb{1}
\end{array}\right), \\
& \boldsymbol{b}=\left(\begin{array}{c}
\boldsymbol{B}_{r}^{(\mathrm{sp})} \\
-\boldsymbol{B}_{r}^{\text {(sp) }}
\end{array}\right), \quad \boldsymbol{f}=\left(\begin{array}{l}
\mathbf{0} \\
\mathbf{1}
\end{array}\right),
\end{aligned}
$$

where $\mathbb{1} \in \mathbb{R}^{N \times N}$ is the identity matrix and $\mathbf{0} \in \mathbb{R}^{D}$ is a vector of zeros.

Sign constraints of the form (17) can be imposed by adding additional rows to matrix $\boldsymbol{A}$ and vector $\boldsymbol{b}$. For every element with sign constraint $B_{r c} \leqslant 0$ on row is added with the entries:

$$
\begin{gathered}
\boldsymbol{A}_{\mathrm{add}}=\left(\boldsymbol{W}_{c, \cdot}, 0, \ldots, 0\right), \\
\boldsymbol{b}_{\text {add }}=-\left[\boldsymbol{B}_{r}^{(\mathrm{sp})}\right]_{c} .
\end{gathered}
$$

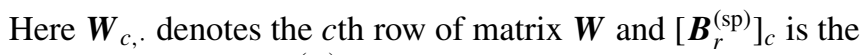
$c$ th entry of vector $B_{r}^{(\mathrm{sp})}$. 
[1] A. P. Sakis Meliopoulos, B. Fardanesh, and S. Zelingher, in Proceedings of the 34th Annual Hawaii International Conference on System Sciences (HICSS-34) (IEEE, New York, 2001), p. 2012.

[2] A. J. Wood, B. F. Wollenberg, and G. B. Sheblé, Power Generation, Operation and Control (Wiley, New York, 2014).

[3] P. Zarco and A. G. Exposito, IEEE Trans. Power Syst. 15, 216 (2000).

[4] K. A. Clements, G. R. Krumpholz, and P. W. Davis, IEEE Trans. Power App. Syst. PAS-100, 1779 (1981).

[5] F. F. Wu and W. H. E. Liu, IEEE Trans. Power Syst. 4, 176 (1989).

[6] D. L. Fletcher and W. O. Stadlin, IEEE Trans. Power App. Syst. PAS-102, 3680 (1983).

[7] W. H. E. Liu, F. F. Wu, and S. M. Lun, IEEE Trans. Power Syst. 7, 81 (1992).

[8] J. Zhu and A. Abur, IEEE Trans. Power Syst. 21, 586 (2006).

[9] L. Zhang and A. Abur, in Proceedings of the 17th Power System Computation Conference, Stockholm, Sweden, Vol. 2226 (2011), p. 788.

[10] A. Abur, H. Kim, and M. K. Celik, IEEE Trans. Power Syst. 10, 2029 (1995).

[11] P. A. Teixeira, S. R. Brammer, W. L. Rutz, W. C. Merritt, and J. L. Salmonsen, IEEE Trans. Power Syst. 7, 1386 (1992).

[12] W. H. E. Liu and S. L. Lim, IEEE Trans. Power Syst. 10, 200 (1995).

[13] O. Alsac, N. Vempati, B. Stott, and A. Monticelli, in Proceedings of the 20th International Conference on Power Industry Computer Applications (IEEE, New York, 1997), p. 90.

[14] P. Zarco and A. Gomez, in Proceedings of the 12th Power System Computation Conference, 1996 (unpublished), p. 1207.

[15] S. Zhong and A. Abur, IEEE Trans. Power Syst. 20, 458 (2005).

[16] A. S. Debs, IEEE Trans. Power App. Syst. PAS-93, 1260 (1974).

[17] I. W. Slutsker, S. Mokhtari, and K. A. Clements, IEEE Trans. Power Syst. 11, 1393 (1996).

[18] M. Ferdowsi, A. Benigni, A. Lowen, B. Zargar, A. Monti, and F. Ponci, IEEE Trans. Instrum. Meas. 64, 1292 (2015).

[19] A. Angioni, J. Shang, F. Ponci, and A. Monti, IEEE Trans. Instrum. Meas. 65, 2234 (2016).

[20] M. K. Yeung, J. Tegner, and J. J. Collins, Proc. Natl. Acad. Sci. U.S.A. 99, 6163 (2002).

[21] T. S. Gardner, D. di Bernardo, D. Lorenz, and J. J. Collins, Science 301, 102 (2003).

[22] M. Timme, Phys. Rev. Lett. 98, 224101 (2007).

[23] D. Napoletani and T. D. Sauer, Phys. Rev. E 77, 026103 (2008).

[24] W.-X. Wang, Y.-C. Lai, C. Grebogi, and J. Ye, Phys. Rev. X 1, 021021 (2011).

[25] R.-Q. Su, W.-X. Wang, and Y.-C. Lai, Phys. Rev. E 85, 065201 (2012).

[26] X. Han, Z. Shen, W.-X. Wang, and Z. Di, Phys. Rev. Lett. 114, 028701 (2015).

[27] L. Ma, X. Han, Z. Shen, W.-X. Wang, and Z. Di, PLoS One 10, e0142837 (2015).

[28] J. Runge, V. Petoukhov, J. F. Donges, J. Hlinka, N. Jajcay, M. Vejmelka, D. Hartman, N. Marwan, M. Paluš, and J. Kurths, Nat. Commun. 6, 8502 (2015).

[29] M. Nitzan, J. Casadiego, and M. Timme, Sci. Adv. 3, 1600396 (2017).

[30] J. Casadiego, M. Nitzan, S. Hallerberg, and M. Timme, Nat. Commun. 8, 2192 (2017).
[31] W. X. Wang, Y. C. Lai, and C. Grebogi, Phys. Rep. 644, 1 (2016).

[32] M. Timme and J. Casadiego, J. Phys. A: Math. Theor. 47, 343001 (2014).

[33] A. Abur and A. G. Exposito, Power System State Estimation: Theory and Implementation (CRC, Boca Raton, 2004).

[34] J. J. Grainger and W. D. Stevenson Jr., Power System Analysis (McGraw-Hill, New York, 1994).

[35] K. Purchala, L. Meeus, D. Van Dommelen, and R. Belmans, in IEEE Power Engineering Society General Meeting, 2005, Vol. 1 (IEEE, New York, 2005), p. 454.

[36] D. Van Hertem, J. Verboomen, K. Purchala, R. Belmans, and W. L. Kling, in 8th IEEE International Conference on AC DC Power Transmission (ACDC 2006) (IET, 2006), pp. 58-62.

[37] M. Newman, Networks: An Introduction (Oxford University, Oxford, 2010), p. 784.

[38] N. Hwang and R. Houghtalen, Fundamentals of Hydraulic Engineering Systems (Prentice-Hall, Upper Saddle River, NJ, 1996).

[39] E. Katifori, G. J. Szöllösi, and M. O. Magnasco, Phys. Rev. Lett. 104, 048704 (2010).

[40] G. B. Giannakis, V. Kekatos, N. Gatsis, S.-J. Kim, H. Zhu, and B. F. Wollenberg, IEEE Signal Process. Mag. 30, 107 (2013).

[41] K. M. Rogers, R. D. Spadoni, and T. J. Overbye, in Power Systems Conference and Exposition (PSCE), 2011 IEEE/PES (IEEE, New York, 2011), p. 1.

[42] K. R. Davis, S. Dutta, T. J. Overbye, and J. Gronquist, in Proceedings of the 46th Hawaii International Conference on System Sciences (HICSS) (IEEE, New York, 2013), p. 2151.

[43] X. Li, H. V. Poor, and A. Scaglione, in Proceedings of the IEEE International Conference on Smart Grid Communications (SmartGridComm) (IEEE, New York, 2013), p. 91.

[44] A. Anwar, A. Mahmood, and M. Pickering, in Proceedings of the IEEE International Conference on Smart Grid Communications (SmartGridComm) (IEEE, New York, 2016), p. 539.

[45] S. Bolognani, N. Bof, D. Michelotti, R. Muraro, and L. Schenato, in Proceedings of the IEEE 52nd Annual Conference on Decision and Control (CDC) (IEEE, New York, 2013), p. 1659.

[46] H. Zhu and G. B. Giannakis, IEEE Trans. Power Syst. 27, 2215 (2012).

[47] S. G. Shandilya and M. Timme, New J. Phys. 13, 013004 (2011).

[48] D. L. Donoho, IEEE Trans. Inf. Theory 52, 1289 (2006).

[49] E. J. Candes, J. K. Romberg, and T. Tao, Commun. Pure Appl. Math. 59, 1207 (2006).

[50] H. Barrios, A. Roehder, H. Natemeyer, and A. Schnettler, in Proceedings of the IEEE PowerTech 2015 Eindhoven (IEEE, New York, 2015), p. 1.

[51] R. D. Zimmerman, C. E. Murillo-Sanchez, and R. J. Thomas, IEEE Trans. Power Syst. 26, 12 (2011).

[52] S. Fliscounakis, P. Panciatici, F. Capitanescu, and L. Wehenkel, IEEE Trans. Power Syst. 28, 4909 (2013).

[53] J. A. Tropp and S. J. Wright, Proc. IEEE 98, 948 (2010).

[54] J. A. Tropp and A. C. Gilbert, IEEE Trans. Inf. Theory 53, 4655 (2007).

[55] C. Soussen, R. Gribonval, J. Idier, and C. Herzet, IEEE Trans. Inf. Theory 59, 3158 (2013).

[56] S. Boyd and L. Vandenberghe, Convex Optimization (Cambridge University, Cambridge, England, 2004). 